\title{
Novel strategies to improve the endocrine therapy of breast cancer
}

\author{
Aurelio Bartolome Castrellon \\ Medical Oncology, Breast Cancer Center, Memorial Cancer Institute, Memorial Healthcare System, Hollywood, FL, USA
}

\begin{abstract}
Endocrine therapy (ET) constitutes the usual first-line of therapy for patients in the treatment of metastatic hormone receptorpositive breast cancer. Unfortunately, not all patients respond to first-line endocrine treatment due to intrinsic resistance, while others may initially respond but eventually progress with secondary acquired resistance leading to disease progression. Mechanisms of resistance to anti-estrogen therapy include, loss of expression for estrogen or progesterone receptor, upregulation of epidermal receptor growth factor 2 , increased receptor tyrosine kinase signaling, leading to activation of various intracellular pathways that are involved in signal transduction such as PI3K/AKT/mammalian target of rapamycin, and others. Growing understanding of the signal cascade of estrogen receptors and the signaling pathways that interact with estrogen receptors has revealed the complex role of these receptors in cell growth and proliferation, and on the mechanism in development of resistance. These insights have led to the development of targeted therapies that may prove to be effective options for the treatment of breast cancer and may overcome hormone therapy resistance. In this review we summarize some of the mechanisms of endocrine resistance, selected clinical trials of ET and targeted therapies, which might interfere with estrogen receptor pathways and might reduce or reverse resistance to traditional, sequential, single-agent ET.
\end{abstract}

\section{Introduction}

The role of estrogen in the growth of breast cancer has been recognized for over a century. George Beatson, a Scottish surgeon, in 1896 described surgical castration as the first systemic therapy for breast cancer. ${ }^{1}$ Beatson was able to recognize the benefits of this approach, even though hormones had not been yet discovered.

Correspondence: Aurelio Bartolome Castrellon, Medical Oncology, Breast Cancer Center, Memorial Cancer Institute, Memorial Healthcare System, Hollywood, FL, USA.

E-mail: acastrellon@mhs.net

Key words: Breast cancer; endocrine therapy.

Received for publication: 4 November 2016.

Accepted for publication: 4 May 2017.

This work is licensed under a Creative Commons Attribution NonCommercial 4.0 License (CC BY-NC 4.0).

(C) Copyright A.B. Castrellon, 2017

Licensee PAGEPress, Italy

Oncology Reviews 2017; 11:323

doi:10.4081/oncol.2017.323
Estrogen mediates its biological effects by binding to $\mathrm{ER} \alpha$ and ER $\beta$, which are members of the nuclear receptor superfamily of ligand-inducible transcription factors. ${ }^{2} \mathrm{ER} \alpha$ is encoded by ESR1, a $300 \mathrm{~kb}$ gene located on chromosome 6 , and has six functional domains, A to F, which include both ligand-binding and DNAbinding domains. ${ }^{3}$ Approximately $75 \%$ of breast cancers express $\mathrm{ER} \alpha$ and belong to the molecular subtypes luminal A or luminal B. ${ }^{4}$ Estrogen receptor positive (ER+) and negative (ER-) disease differ in terms of clinical behavior, prognosis, patterns of recurrence, and aggressiveness. Patients with ER+ disease are likely to have more indolent disease, bone metastases, and late recurrences. ${ }^{5}$

Given their proven efficacy and generally favorable toxicity profile, with the exception of patients with advanced visceral disease, most patients will receive endocrine therapies (ET) in the treatment of metastatic ER+ breast cancer (BC). In premenopausal women, tamoxifen, pharmacological or surgical ovarian ablation are standard, while in postmenopausal women, aromatase inhibitors (AI) are prescribed to block the conversion of weak androgens of adrenal origin to estrogen in peripheral tissues as well as breast cancer tissue itself. ${ }^{6}$ Fulvestrant is an ER downregulator and a more potent antiestrogen that reduces ER levels in cells. ${ }^{7}$ Unfortunately, not all patients respond to first-line ET due to intrinsic resistance, while others may initially respond but eventually progress with secondary acquired resistance leading to disease progression and endocrine resistance. ${ }^{8}$ The response to second line ET has a tendency to be brief, as demonstrated in clinical trials including, the EFECT (Evaluation of Faslodex versus Exemestane Clinical Trial), SoFEA (Study Of Faslodex with or without concomitant Arimidex vs Exemestane following progression on non-steroidal aromatase inhibitors) with progression free survival (PFS) of around 4-5 months in all study groups. ${ }^{9,10}$

Mechanisms of resistance to anti-estrogen therapy include, ER loss over time in the tumor which occurs in about $20 \%$ of patients treated with ET, acquired mutations in ER $\alpha$ (ESR 1), constitutive activation cyclin-dependent kinases (CDK) 4 and $6 .{ }^{8}$ Upregulation of epidermal receptor growth factor 2 (HER2) by either acquisition of gene amplification or overexpression, HER2 may subsequently assume the driving role in tumor progression by serving as an alternative survival pathway or by reducing the level of ER, thus rendering the tumor less responsive to estrogen. ${ }^{11,12}$ Progesterone receptor (PR) is lost more frequently than ER while patients undergo ET. This loss leads to tumor aggressiveness and worse survival outcome than patients who maintain PR expression after resistance to ET. ${ }^{13}$ Other mechanisms of resistance are associated with increased receptor tyrosine kinase signaling, leading to activation of various intracellular pathways involved in signal transduction, such as $\mathrm{PI} 3 \mathrm{~K} / \mathrm{AKT} / \mathrm{mammalian}$ target of rapamycin (mTOR), including loss of phosphatase and tensin homolog (PTEN) function, PI3K mutations, aberrant activation of AKT, mitogen activated kinase (MAPK)/ERK, fibroblast growth factor receptor and insulin-like growth factor-1 receptor. ${ }^{14-19}$ Also described are alterations in cell cycle and apoptotic machinery; Epigenetic modification including dysregulation of DNA methy- 
lation, histone modification, nucleosome remodeling and altered expression of specific microRNAs. ${ }^{20,21}$

Over the past few years a number of clinical trials have tested the addition of novel therapeutic agents to standard ET, with the purpose of improving the response and preventing the resistance to ET. This has resulted in the approval of three agents; the mTOR inhibitor everolimus and two CDK 4/6 inhibitors: palbociclib and ribociclib. Current ongoing studies will likely further improve the drug armamentarium for the treatment of locally advanced and metastatic breast cancer (MBC). In this review we summarize some of the clinical trials that have seeked to improve the response of ET in breast cancer by combining drugs that can help overcome intrinsic resistance of tumor and affect pathways associated with poor response to ET.

\section{mTOR inhibitors}

The PI3K-AKT (a serine/threonine kinase) pathway plays a central role in cell survival, proliferation and angiogenesis and is frequently deregulated in cancer. ${ }^{22-24}$ A close interaction between the mTOR pathway and ER signaling has been reported. A substrate of the mTOR complex 1, S6 kinase 1 phosphorylates the activation function domain 1 of the ER, leading to ligand-independent receptor activation. Estrogen-dependent cells, cultured long term in estrogen-depleted medium, rely on mTOR signaling for growth and are excessively sensitive to its inhibition. ${ }^{25}$ In addition, mTOR inhibition restores sensitivity of endocrine-resistant breast cancer cells to endocrine therapy. ${ }^{26,27}$

Prior to reports from m-TOR inhibitors studies in the metastatic setting (Table 1), ${ }^{28-33}$ a phase II neoadjuvant endocrine therapy clinical trial of breast cancer, demonstrated synergistic effect to the combination of everolimus with letrozole. In this study, 270 postmenopausal women with operable ER+/HER2 negative breast cancer were randomly assigned to receive 4 months of neoadjuvant treatment with letrozole and either everolimus $(10 \mathrm{mg} /$ day $)$ or placebo. The primary endpoint, response rate by clinical examination, was significantly improved from $59.1 \%$ to $68.1 \%$ in the combined treatment arm $(\mathrm{P}=0.062)$. The patients had mandatory biopsies at baseline and at day 15, allowing key biomarker analyses as a secondary endpoint. Downregulation of phospho-S6 and reduction in Ki67 expression was more frequently seen in the everolimus arm. ${ }^{28}$
Although active in renal cancer, the m-TOR inhibitor temsirolimus was not found to be effective in the treatment of advanced breast cancer. In the phase III HORIZON clinical trial 1112 postmenopausal patients with MBC were randomized to receive front line therapy with either letrozole daily combined with temsirolimus $30 \mathrm{mg}$ intermittent daily ( 5 days every 2 weeks) or letrozole and placebo. The study was prematurely closed for futility at the preplanned second interim analysis with a PFS of 9 and 8.9 months respectively. ${ }^{29}$ The disappointing results of this large randomized trial were followed by positive findings in a much smaller open-labeled phase II study. The TAMRAD clinical trial randomized 111 postmenopausal patients following progression to AI to receive tamoxifen $20 \mathrm{mg}$ oral daily combined with everolimus or tamoxifen alone. The primary endpoint was clinical benefit rate (CBR) defined as objective response or stable disease for $\geq 24$ weeks according to RECIST v 1.0 (Response Evaluation Criteria In Solid Tumors version 1.0). The CBR was higher in the combined treatment arm (61\% versus $42 \%)$ and the time to progression (TTP) was increased from 4.5 months to 8.6 months favoring the investigational arm (hazard ratio [HR], 0.54; 95\% CI, 0.36 to 0.81 ). The main toxicities were more common in the tamoxifen plus everolimus arm and included, fatigue $(72 \% v s$ $53 \%$ ) stomatitis ( $56 \%$ vs $7 \%$ ), rash (44\% vs 7\%), anorexia (43\% vs $18 \%)$, and diarrhea ( $39 \%$ vs 11$).{ }^{30}$

The encouraging results from the TAMRAD study led to the design of the randomized phase III BOLERO-2 clinical trial. In this study 724 postmenopausal HR+/HER2 negative patients with advanced $\mathrm{BC}$ were randomized in a 2:1 ratio favoring the investigational arm to receive either everolimus at the dose of $10 \mathrm{mg}$ daily in combination with exemestane or exemestane and placebo. All patients suffered from recurrence of breast cancer 12 months after the end of adjuvant treatment or during treatment for MBC with either anastrozole or letrozole. Other previous anticancer endocrine treatments and a single prior chemotherapy regimen for advanced disease were also allowed. The trial met the primary end point, PFS; the median PFS, on the basis of central assessment were 10.6 months and 4.1 months, respectively favoring the everolimus containing arm $(\mathrm{P}<0.001) .{ }^{31}$ Based on results of this phase III clinical trial everolimus is currently approved for the treatment of ER+, HER2 negative advanced breast cancer in combination with exemestane in patients resistant to nonsteroidal aromatase inhibitors.

The use of everolimus in the frontline setting of $\mathrm{MBC}$ is being evaluated in the phase 2 BOLERO-4 trial, where 202

Table 1. Selected clinical trials of $\mathrm{m}$-TOR inhibitors + ET.

\begin{tabular}{|c|c|c|c|c|}
\hline Study & Design & Drug combination & $\mathbf{N}$ & Results \\
\hline Baselga et al..$^{28}$ & $\begin{array}{l}\text { Phase II } \\
\text { Neoadjuvant }\end{array}$ & Letrozole-everolimus & 270 & $\begin{array}{l}\text { RR by clinical examination, } \\
59.1 \% \text { to } 68.1 \%\end{array}$ \\
\hline TAMRAD ${ }^{30}$ & $\begin{array}{c}\text { Phase II } \\
\text { MBC pretreated }\end{array}$ & Tamoxifen-everolimus vs tamoxifen & 111 & TTP increased from 4.5 to $8.6 \mathrm{~m}$ \\
\hline $\mathrm{HORIZON}^{29}$ & $\begin{array}{c}\text { Phase III } \\
\text { MBC } 1^{\text {st }} \text { line }\end{array}$ & Letrozole-temsirolimus vs letrozole & 1112 & No improvement in PFS 9 vs $8.9 \mathrm{~m}$ \\
\hline BOLERO-2 $2^{31}$ & $\begin{array}{c}\text { Phase III } \\
\text { MBC pretreated }\end{array}$ & Exemestane-everolimus vs exemestane & 724 & Increase in PFS from 4.1 to $10.6 \mathrm{~m}$ \\
\hline BOLERO-4 ${ }^{32}$ & $\begin{array}{c}\text { Phase II } \\
\text { MBC } 1^{\text {st }} \text { line }\end{array}$ & Letrozole-everolimus vs letrozole & 202 & Median PFS not reached at $17.5 \mathrm{~m}$ \\
\hline PrECOG $0102^{33}$ & $\begin{array}{c}\text { Phase II } \\
\text { MBC pretreated }\end{array}$ & Fulvestrant-everolimus vs fulvestrant & 131 & Increase in PFS 10.4 to $5.1 \mathrm{~m}$ \\
\hline
\end{tabular}


Postmenopausal patients with HR+/HER2 negative metastatic or locally advanced $\mathrm{BC}$ with no prior advanced disease therapy received everolimus at the dose of $10 \mathrm{mg}$ /day combined with letrozole. At disease progression, patients were offered everolimus and exemestane $25 \mathrm{mg} /$ day until further progression or unacceptable toxicity. Patients with stomatitis completed the Oral Stomatitis Daily Questionnaire and were randomized to local standard of care or alcohol-free dexamethasone $0.5 \mathrm{mg} / 5 \mathrm{~mL}$ oral rinse, where commercially available. Median PFS has not been reached at the median follow-up of 17.5 months. Researchers estimated the 6month and 12-month PFS rates to be $83.6 \%$ (95\% CI, 77.3-88.2) and $71.4 \%$ (95\% CI, 64.0-77.5), respectively. The overall response rate was $42.6 \%$ (95\% CI, 35.7-49.7) the CBR for the combination was $74.3 \%(95 \% \mathrm{CI}, 67.7-80.1)$. $^{32}$

PrECOG 0102 sought to evaluate the combination of everolimus with fulvestrant vs fulvestrant plus placebo in 131 postmenopausal women HR+/HER2 negative inoperable $\mathrm{BC}$ (locally advanced or metastatic) previously treated with an AI for metastatic disease or relapsing on adjuvant AI. One prior chemotherapy regimen for metastatic disease was allowed. Patients were randomized in a 1:1 ratio in a blinded manner to fulvestrant $(500 \mathrm{mg} / \mathrm{d}$ on days 1 and 15 of cycle 1 , then day 1 of cycles $2-12$ given every 28 days $)$ plus oral everolimus $(10 \mathrm{mg} / \mathrm{d})$ or the same dose of fulvestrant plus placebo during the induction phase. Treatment continued until disease progression or unacceptable toxicity, for a maximum of 12 cycles (48 weeks). The study met the primary endpoint for PFS by investigator assessment, patients receiving the combination of fulvestrant and everolimus had a significant improvement in PFS of 10.4 versus 5.1 months for the group receiving fulvestrant/placebo. The hazard ratio was $0.60(\mathrm{P}=0.02){ }^{33}$ There was no difference in OS, the combination was associated with more toxicity, including more frequent known everolimus therapy grade 3 adverse events.

\section{Anti-HER 2 therapy}

Prior to establishing the standard of care for ER+/HER2 negative $\mathrm{MBC}$, the combination of a taxane with trastuzumab and pertuzumab. ${ }^{34}$ Clinical trials evaluated the feasibility and therapeutic effect of combining ET with anti-HER 2 agents (Table 2). ${ }^{35,36}$ The first of these studies the TAnDEM phase III clinical trial, included 207 postmenopausal patients with $\mathrm{HR}+$ /HER 2 positive MBC, who were randomly assigned to received anastrozole alone $(n=104)$ or trastuzumab plus anastrozole (103). Patients in the trastuzumab plus anastrozole arm experienced a significant improvements in PFS of 4.8 months compared with patients receiving anastrozole alone 2.4 months $(\mathrm{P}=0.0016) .{ }^{35}$ Similarly a phase III clinical trial randomized 1286 postmenopausal patients with either HR+/HER2 positive or HR+/HER2 negative MBC to receive letrozole plus lapatinib vs letrozole plus placebo. In the HR+/HER2 positive patients $(n=219)$, addition of lapatinib to letrozole significantly reduced the risk of disease progression with a median PFS of 8.2 months in comparison to 3.0 months in the letrozole-placebo group ( $\mathrm{P}=0.019)$. No improvement in PFS was observed among patients with HER2 negative disease. ${ }^{36}$

These two studies highlight the fact that the PFS in patients with $\mathrm{HR}+/ \mathrm{HER} 2$ positive MBC treated with endocrine therapy alone is very brief when compared to HR+/HER2 negative. ${ }^{6}$ Also, that despite the fact that the addition of anti-HER2 therapy to ET is effective, results are inferior to what is seen with chemotherapy and anti-HER2 therapy. ${ }^{34}$ These findings are the reason why endocrine therapy in combination with anti-HER2 therapy is reserved for patients with very indolent disease or in those who are not candidates for chemotherapy. ${ }^{37}$

\section{Inhibition of the proteasome}

The proteasome is a multi-catalytic, multi-subunit protease complex that is responsible for the ubiquitin-dependent turnover of cellular proteins. ${ }^{38}$ The proteolytic component of this system, the $26 \mathrm{~S}$ proteasome, consists of two $19 \mathrm{~S}$ regulatory particles, involved in substrate recognition and unfolding and a core particle, the $20 \mathrm{~S}$ proteasome. Since proteasomes play a central role in the cytoplasmic turnover of the vast majority of proteins, the manipulation of proteasomal activity is a key goal in controlling the stability of regulatory proteins. ${ }^{39}$ Inhibition of the proteasome results in abnormal accumulation of many intracellular proteins, thereby disrupting cellular homeostasis and results in the induction of tumor cell apoptosis. $^{40}$

Preclinical studies have demonstrated that proteasome inhibition might be a potential therapeutic tool for the treatment of endocrine resistant breast cancer. Mechanisms of this effect include, inhibition of mitogen activated protein kinase phosphatase-1 (MKP-1), ${ }^{41}$ and inhibition of signaling cascades that are key regulators of hormone independence and anti-endocrine resistance. ${ }^{42-44}$

The combination of fulvestrant and bortezomib has been studied in a group of postmenopausal women, who had experienced progressive disease following AI therapy. The study randomized 118 patients to receive fulvestrant alone at the dose of $500 \mathrm{mg}$ or in combination with bortezomib $\left(1.6 \mathrm{mg} / \mathrm{m}^{2} \mathrm{IV}\right.$ on days $\left.1,8,15\right)$. The primary endpoint was PFS. At 12 months, the PFS was $13.6 \%$ for the fulvestrant alone group $v s 28.1 \%$ in patients treated with fulvestrant and bortezomib $(\mathrm{P}=0.03) .{ }^{45}$ This study indicates that proteasome inhibitors could have some activity delaying progression to ET and perhaps further studies should be performed evaluating these agents.

\section{Histone deacetylase inhibitors}

Histone acetylation is an important determinant of gene expression. Acetylation is generally associated with elevated transcription, while deacetylated histones are often associated with

Table 2. Selected clinical trials of anti-HER2 + endocrine therapy.

\begin{tabular}{|c|c|c|c|c|}
\hline Study & Design & Drug combination & $\mathbf{N}$ & Results \\
\hline TAnDEM $^{35}$ & $\begin{array}{c}\text { Phase III } \\
\text { MBC } 1^{\text {st }} \text { line }\end{array}$ & Anastrozole-trastuzumab vs anastrozole & 207 & Increase in PFS 2.4 vs $4.8 \mathrm{~m}$ \\
\hline Johnston et al. ${ }^{36}$ & $\begin{array}{c}\text { Phase III } \\
\text { MBC } 1^{\text {st }} \text { line }\end{array}$ & Letrozole-lapatinib vs letrozole & 219 & Increase in PFS 3.0 vs $8.2 \mathrm{~m}$ \\
\hline
\end{tabular}

$\mathrm{m}$, months; PFS, progression free survival; MBC, metastatic breast cancer. 
gene repression. ${ }^{46}$ Histone deacetylases (HDACs) are critical regulators of gene expression that enzymatically remove the acetyl group from histones. With HDAC inhibition, epigenetic changes may lead to the loss of ER $\alpha$ and make it more difficult to inhibit this receptor. ${ }^{47} \mathrm{~A}$ possible approach to reverse hormone resistance in the treatment of breast cancer, is the use of histone deacetylase inhibitors (HDACI) to re-sensitize breast cancer cells to hormone manipulation. ${ }^{48}$ Entinostat is an HDACI that has been shown to increase expression of both ER and the enzyme aromatase in a dose-dependent manner both in vitro and in vivo, which then sensitized breast cancer cells to estrogen and subsequent inhibition by the AI letrozole. ${ }^{49}$

The combination of the AI exemestane with entinostat was evaluated in the phase II ECORE 301 clinical trial. In this study 130 postmenopausal patients with history of advanced breast cancer that had progressed on a non-steroidal AI were randomized to receive exemestane with entinostat $5 \mathrm{mg}$ or placebo weekly. $33 \%$ of the patients had received chemotherapy in the advanced breast cancer setting. Although small, this trial did demonstrate a statistically significant improvement in the PFS favoring the investigational arm (4.28 vs 2.27 months). The most striking findings of the trial was that the addition of entinostat led to a 10 month improvement in overall survival, from 19.8 months with exemestane alone to 28.1 months with the combination $(\mathrm{P}=0.04) .{ }^{50}$ Correlative studies suggest that HDAC2 expression could be a predictive biomarker, and that histone hyper-acetylation may be a valid pharmacodynamic marker for the efficacy of this combination. ${ }^{51}$ The phase III E2112 trial will further evaluate entinostat plus exemestane in 600 metastatic patients. Importantly, prior treatment with everolimus is allowed in this study (NCT02115282).

\section{Antiangiogenic agents}

High vascular endothelial growth factor (VEGF) levels in breast tumors have been associated with a decreased response to ET. ${ }^{52,53}$ The feasibility and activity of the VEGF inhibitor bevacizumab in combination with endocrine agents had been previously tested in a phase II clinical trial with encouraging results. ${ }^{54}$ Based on these findings, the phase III randomized LEA clinical trial evaluated bevacizumab with ET in the first line setting of postmenopausal patients with HR+/HER2 negative MBC. In this study 380 patients were randomly assigned to receive ET alone or in combination with bevacizumab. Of the patients, 342 received letrozole and 38 fulvestrant. Although PFS in this study was increased to 18.4 months with the addition of bevacizumab vs 13.4 without, it did not reach statistical significance $(\mathrm{P}=0.14)$. The combination had a significantly higher incidence of hematologic and non-hematologic toxicities therefore does not appear to be a promising approach to enhance first line therapy. ${ }^{55}$

\section{Agents targeting the FGFR pathway}

Fibroblast growth factors (FGFs) are involved in cancer cell proliferation and new blood vessel formation. FGFs are a family of related extracellular proteins that normally regulate cell proliferation and survival in humans. ${ }^{56}$ They act by binding to and activating FGF receptors (FGFRs), which are cell surface proteins that transmit growth signals to cells. Certain FGFs promote growth of multiple solid tumors by binding and activating FGFRs. ${ }^{57}$ The FGF family consists of 22 known proteins called ligands that exert their physiological effect on cells by binding to four FGFRs
(FGFR1, 2, 3 and 4). ${ }^{58}$

In addition to FGFR1 gene amplification, certain tumors contain an excessive number of gene copies encoding FGF ligands 3, 4 and 19. Because these genes are located together on chromosome 11, amplification of FGF 3, 4 and 19 is commonly referred to as $11 \mathrm{q}$ amplification. The amplification of these genes in the tumor cell has the potential to increase FGFR activation and tumor growth. ${ }^{59}$

The FGFR1 and/or 11q gene amplification has been observed in up to $25 \%$ of breast malignant tumors. ${ }^{60}$ Several studies are evaluating FGFR inhibitors in the treatment of breast cancer with or without antiestrogen therapies. ${ }^{61-64}$ Dovitinib (TKI258) a small molecule inhibitor of FGFR1-3, VEGFR1-3, c-KIT, fms-related tyrosine kinase 3 (FLT3), platelet-derived growth factor receptor (PDGFR) $\beta$, c-KIT, and FLT3, is being evaluated in combination with fulvestrant in postmenopausal patients with locally advanced or metastatic HER2 negative, HR breast cancer who have progressed during or following endocrine therapy. ${ }^{63}$ Lucitanib (E3810) a small molecule inhibitor of VEGFR1-3, FGFR1-2, and colony stimulating factor 1 receptor has demonstrated anti-angiogenic and anti-tumor activity in preclinical models. A phase 2 study of lucitanib is ongoing in patients with ER + FGFR1-amplified and non-amplified metastatic breast cancer (NCT02053636).

\section{Inhibitors of cyclin-dependent kinase 4/6}

Cell cycle regulation is identified as an attractive target for targeted drug therapy. Inhibitors of cyclin-dependent kinase (CDK) 4 and 6, together with cyclin D, promote G1-to-S phase transition by phosphorylating the retinoblastoma protein, which releases the E2F transcription factor and activates downstream target genes. ${ }^{64}$ CDK4/6 is particularly activated in ER+ breast cancer via the ER, along with other oncogenic signaling pathways. ${ }^{65}$ Given their kinase activity, the cyclin dependent kinases have been pursued as drug targets. ${ }^{66}$ After disappointing results of first and second generation CDK inhibitors mainly due to low single agent efficacy and increased toxicity, ${ }^{67}$ the development of specific CDK 4/6 inhibitors has produced results never before seen in the treatment of breast cancer ${ }^{68}$ (Table 3). ${ }^{69-77}$

Palbociclib was the first of these agents to gain approval, it did so by demonstrating activity in the phase II open label randomized PALOMA 1 clinical trial. In this study, 165 postmenopausal women with advanced ER+ and HER2 negative breast cancer, who had not received any systemic treatment for their advanced disease were randomly assigned in a 1:1 to receive continuous letrozole or letrozole daily plus oral palbociclib $125 \mathrm{mg}$, given once daily for 3 weeks followed by 1 week off over 28 -day cycles. The major efficacy outcome measure was investigator-assessed PFS of 10.2 months for the letrozole group and 20.2 months for the palbociclib plus letrozole group $(\mathrm{P}=0.0004) .{ }^{69}$ These results were confirmed in the larger phase III PALOMA-2 clinical trial. This study randomized 666 patients 2:1 to receive the same dose and frequency of letrozole or letrozole plus palbociclib. Patients in the palbociclib containing arm experienced a PFS of 24.8 months in comparison to the control arm of 14.5 months $(\mathrm{P}<0.000001)$. Over all response rate (ORR) was also higher with palbociclib with $55.3 \%$ of patients who had measurable disease experiencing a reduction in size $v s$ $44 \%(\mathrm{P}=0.013)$. In terms of side effects, neutropenia (79.5 vs $6.3 \%)$ and fatigue (37.4 vs $27.5 \%$ ) were more noticeable in the investigational arm; neutropenic fever was only seen in $2.5 \%$ of the patients. ${ }^{70}$

Similarly, in the second line setting the PALOMA-3 doubleblind phase III clinical trial, randomized 427 patients whose dis- 
ease had progressed within 12 months of adjuvant therapy or within one month of endocrine therapy for HR+/HER2 negative MBC to receive palbociclib plus fulvestrant versus fulvestrant plus placebo. In conjunction with study treatment, premenopausal and perimenopausal women were required to take goserelin. The study met the primary endpoint, PFS which was 9.2 months in the palbociclib and fulvestrant arm versus 3.8 months in the fulvestrant and placebo arm $(\mathrm{P}<0.001) .{ }^{71}$

In the neoadjuvant setting, a phase II clinical trial evaluated the early introduction of CDK 4/6 inhibitors in the treatment of breast cancer. This is based on the observation that decreasing Ki 67 by complete cell cycle arrest, could have a positive effect on long term outcomes. ${ }^{72}$ The study included patients with clinical stage II/III HR+, HER2 negative breast cancer. Patients received palbociclib and anastrozole for a four-month period and a proportion of patients was kept on palbociclib up until surgery; serial biopsies were performed. The primary end point was complete cell-cycle arrest, which was defined as a proportion of tumor cells positive for $\mathrm{Ki} 67 \leq 2.7 \%$ on cycle 1 , day 15 after 2 weeks of treatment with both drugs. Of the 45 evaluable patients $87 \%$ experienced complete cell-cycle arrest at cycle 1, day 15. Clinical responses were observed in $67 \%$. Patients tended to have a rebound in Ki67 level in the washout period; however, this increase was not observed in patients who continued palbociclib. ${ }^{73}$ The neoMONARCH phase II trial evaluated abemaciclib in combination with anastrozole in the neoadjuvant setting. In this study 173 women were randomized to receive abemaciclib plus anastrozole $(n=56)$, abemaciclib monotherapy $(n=58)$ or anastrozole monotherapy $(n=59)$ for the first two weeks. At the conclusion of that regimen, all patients underwent a second core biopsy and then subsequently received the abemaciclib-anastrozole combination for 14 weeks. Abemaciclib was administered in $150 \mathrm{mg}$ oral doses every $12 \mathrm{~h}$, and anastrozole was administered in $1 \mathrm{mg}$ oral doses daily. Patients also received loperamide as primary prophylaxis with each abemaciclib dose. The percentage of Ki67 responders, defined as patients with Ki67 levels $<2.7 \%$ at week 2, was higher among those assigned the combination (69.6\%) and abemaciclib monotherapy (68.4\%) than anastrozole alone $(22.7 \%)$, radiographic response rate (RR) was $54.7 \% .^{74}$

The results for ribociclib were reported in the MONALEESA2 Phase III randomized, double blind, placebo controlled, and multicenter global registration trial. The study randomized 668 post- menopausal women with HR+/HER2 negative advanced breast cancer in a 1:1 stratified by the presence of liver and/or lung metastases. Patients received ribociclib $600 \mathrm{mg}$ /daily (three weeks on and one week off), or placebo, in combination with letrozole 2.5 $\mathrm{mg} /$ daily. The first interim analysis showed a $44 \%$ improvement in median PFS and has not been yet reached at the data cut-off over 14.7 months seen in the placebo arm $(\mathrm{P}=0.00000329)$. As seen with other CDK4/6 inhibitors there is a significantly higher objective response rate when combined with AIs (53\% vs 37\%; $\mathrm{P}=0.00028)$. This agent is also associated with neutropenia which occurred in $59 \%$ of patients in the ribociclib arm compared to $1 \%$ of the placebo arm; leukopenia occurred in $21 \%$ vs $1 \%$. ${ }^{75,76}$ The MONALEESA-3 trial is evaluating ribociclib in combination with fulvestrant compared to fulvestrant alone in men and postmenopausal women with HR+/HER2 negative MBC in the second line endocrine therapy setting (NCT02422615).

Abemaciclib has demonstrated single agent activity as reported in the MONARCH 1 phase II single arm study where 132 patients received abemaciclib monotherapy $200 \mathrm{mg}$ every 12 hours until progression of disease. Patients had a median of 3 lines of prior therapy for advanced disease, including a median of 2 lines of chemotherapy for advanced disease, $90.2 \%$ had visceral disease. At the 8 month interim the confirmed ORR (per RECIST v1.1) was $17.4 \%$, the CBR defined as objective response or stable disease for $\geq 24$ weeks was $42.4 \%$, and median PFS was 5.7 months. ${ }^{77}$ Two additional MONARCH trials are evaluating abemaciclib in breast cancer. MONARCH 3 is a Phase III trial of abemaciclib in combination with anastrozole in patients with $\mathrm{HR}+$ /HER2 negative locoregionally recurrent or metastatic breast cancer (NCT02246621). The monarcHER is evaluating abemaciclib plus trastuzumab (with or without fulvestrant) in women with HR+/HER2 positive locally advanced or metastatic breast cancer (NCT02675231).

\section{Fulvestrant in the first line setting}

Fulvestrant was initially approved at the dose of $250 \mathrm{mg}$ following progression on an antiestrogen therapy, such as tamoxifen. However, pharmacokinetic findings from the phase III EFECT trial prompted researchers to explore a $500 \mathrm{mg}$ dose. ${ }^{9}$ Two clinical trials

Table 3. Selected clinical trials of CDK 4/6 inhibitors.

\begin{tabular}{|c|c|c|c|c|}
\hline Study & Design & Drug combination & $\mathbf{N}$ & Results \\
\hline Paloma $1^{69}$ & $\begin{array}{l}\text { Phase II } \\
\text { MBC } 1^{\text {st }} \text { line }\end{array}$ & Letrozole-palbociclib vs letrozole & 165 & Improvement in PFS from 10.2 to $20.2 \mathrm{~m}$ \\
\hline Paloma $2^{70}$ & $\begin{array}{l}\text { Phase III } \\
\text { MBC } 1^{\text {st line }}\end{array}$ & Letrozole-palbociclib $v$ s letrozole & 666 & Improvement in PFS from 14.5 to $24.8 \mathrm{~m}$ \\
\hline Paloma $3^{71}$ & $\begin{array}{c}\text { Phase III } \\
\text { MBC pretreated }\end{array}$ & Fulvestrant palbociclib $v s$ fulvestrant & 427 & Improvement in PFS from 3.8 to $9.2 \mathrm{~m}$ \\
\hline Ma et al..$^{73}$ & $\begin{array}{c}\text { Phase II } \\
\text { Neoadjuvant }\end{array}$ & Anastrozole-palbociclib & 45 & $\begin{array}{l}\text { 87\% complete cell-cycle arrest at cycle } 1 \text {, } \\
\text { day } 15 \text {. Clinical RR } 67 \%\end{array}$ \\
\hline MONALEESA-276 & $\begin{array}{l}\text { Phase III } \\
\text { MBC } 1^{\text {st }} \text { line }\end{array}$ & Letrozole-ribociclib vs letrozole & 668 & $\begin{array}{l}\text { 44\% improvement in PFS } 14.7 \mathrm{~m} v \mathrm{~s} \\
\text { NR for ribociclib }\end{array}$ \\
\hline MONARCH-177 & $\begin{array}{c}\text { Phase II } \\
\text { MBC (heavily-pretreated) }\end{array}$ & Abemaciclib & 132 & ORR 17.4\%, CB 42.4\%, PFS $5.7 \mathrm{~m}$ \\
\hline neoMONARCH ${ }^{74}$ & $\begin{array}{c}\text { Phase II } \\
\text { Neoadjuvant }\end{array}$ & $\begin{array}{l}\text { Abemaciclib + anastrozole } v s \\
\text { abemaciclib } v s \text { anastrozole }\end{array}$ & 173 & $\begin{array}{c}\text { Ki67 <2.7\% at week 2. Combination (69.6\%); } \\
\text { abemaciclib (68.4\%); anastrozole (22.7\%). } \\
\text { RR 54.7\% }\end{array}$ \\
\hline
\end{tabular}

m, months; PFS, progression free survival; TTP, time to progression; ORR, over all response rate; RR, response rate; CB, clinical benefit. 
have explored fulvestrant in the front line treatment of MBC. The FIRST phase II open-label study randomized postmenopausal patients with ER +/HER2 negative MBC to receive fulvestrant $(n=102)$ or anastrozole $(n=103)$. The study allowed for prior adjuvant endocrine therapy. The primary endpoint of the study was CBR, defined as objective response or stable disease for $\geq 24$ weeks. $72.5 \%$ CBR was observed with fulvestrant compared with $67 \%$ with anastrozole $(\mathrm{P}=0.386)$. The study demonstrated an impressive TTP of 23.4 versus 13.1 months favoring fulvestrant $(\mathrm{P}=0.01) .{ }^{78}$ OS was also improved to 54.1 months with fulvestrant compared with 48.4 months with anastrozole $(\mathrm{P}=0.041) .{ }^{79}$ The confirmatory FALCON phase III clinical trial randomized 462 postmenopausal patients with advanced breast cancer in a $1: 1$ to receive the same dose and frequency of fulvestrant or anastrozole. The study met the primary end point PFS. Patients receiving fulvestrant had a PFS of 16.6 vs 13.8 months with anastrozole $(\mathrm{P}=0.0486) .{ }^{80}$

\section{Conclusions}

ET is central for many women with breast cancer, but resistance to therapy unavoidably occurs. The ER signaling pathway is a composite network of extensive crosstalk with growth-factor signaling pathways, cell cycle control pathways, and protein degradation pathways. These pathways provide many alternative targets for agents that may be useful in combination with ET to decrease resistance to treatment and to extend benefit to patients who do not achieve optimal benefit from ET alone. Aromatase inhibitors have been for decades the preferred front line therapy for MBC patients who are not experiencing a visceral crisis, fulvestrant has been reserved for the second line setting. Recent studies demonstrating improved results with first-line therapy fulvestrant indicate this agent can be considered in this setting. With the development of CDK 4/6 inhibitors significant improvement in PFS has been documented in each trial, where these agents have been combined with antiestrogen therapies. It is however, unknown if there is benefit to the continuation of these agents following progression. There is an ongoing studies looking into combining everolimus with ribociclib, following progression in the first-line ET in combination with a CDK 4/6 inhibitor (NCT02732119). Everolimus will likely continue to be used following progression to a CDK 4/6 inhibitor in combination with exemestane or fulvestrant. The toxicity profile of the CDK 4/6 inhibitors although favorable, caution should be taken in to adjusting the dose of these agents adequately as severe neutropenia and other side effects have been reported. With the broader use of molecular profiling, identifying patients with mutations in the FGFR could improve the participation of patients in clinical trials of these agents, with promising potential in the treatment of MBC.

The future looks very promising for the ET of patients with $\mathrm{MBC}$, with unprecedented PFS findings on recent trials, it is likely that the overall survival of patients will continue to improve overtime. Part of the success of these agents is in overcoming intrinsic resistance of cancer and preventing acquired resistance over time. The question remains however, on which patients are these drug combinations needed, as adding these agents to ET increases toxicity and cost. Biomarkers that predict the benefit of these targeted therapies are greatly needed.

\section{References}

1. Beatson, GT. On the treatment of inoperable cases of carcinoma of the mamma: suggestions for a new method of treatment, with illustrative cases. Lancet 1896;2:104-7.

2. Gross JM, Yee D. How does the estrogen receptor work? Breast Cancer Res 2002;4:62-4.

3. Kumar V, Green S, Stack G, et al. Functional domains of the human estrogen receptor. Cell 1987;51:941-51.

4. Levin ER. Integration of the extranuclear and nuclear actions of estrogen. Mol Endocrinol 2005;19:1951-9.

5. Blanco G, Holli K, Heikkinen M, et al. Prognostic factors in recurrent breast cancer: relationships to site of recurrence, disease-free interval, female sex steroid receptors, ploidy and histological malignancy grading. Br J Cancer 1990;62:142-6.

6. Lumachi F, Luisetto G, Basso SM, et al. Endocrine therapy of breast cancer. Curr Med Chem 2011;18:513-22.

7. Croxtall JD, McKeage K. Fulvestrant: a review of its use in the management of hormone receptor-positive metastatic breast cancer in postmenopausal women. Drugs 2011;71:363-80.

8. Osborne CK, Schiff R. Mechanisms of endocrine resistance in breast cancer. Annu Rev Med 2011;62:233-47.

9. Chia S, Gradishar W, Mauriac L, et al. Double-blind, randomized placebo controlled trial of fulvestrant compared with exemestane after prior nonsteroidal aromatase inhibitor therapy in postmenopausal women with hormone receptor-positive, advanced breast cancer: results from EFECT. J Clin Oncol 2008;26:1664-70.

10. Johnson S, Kilburn LS, Ellis P, et al. Fulvestrant plus anastrozole or placebo versus exemestane alone after progression on non-steroidal aromatase inhibitors in postmenopausal patients with hormone-receptor-positive locally advanced or metastatic breast cancer (SoFEA): a composite, multicenter, phase 3 randomized trial. Lancet Oncol 2013;14:989-98.

11. Knowlden JM, Hutcheson IR, Jones HE, et al. Elevated levels of epidermal growth factor receptor/c-erbB2 heterodimers mediate an autocrine growth regulatory pathway in tamoxifen resistant MCF-7 cells. Endocrinology 2003;144:1032-44.

12. Kurokawa H, Lenferink AE, Simpson JF, et al. Inhibition of HER2/neu (erbB-2) and mitogenactivated protein kinases enhances tamoxifen action against HER2-overexpressing, tamoxifen-resistant breast cancer cells. Cancer Research 2000;60:5887-94.

13. Cui X, Schiff R, Arpino G, et al. Biology of progesterone receptor loss in breast cancer and its implications for endocrine therapy. J Clin Oncol 2005;23:7721-35.

14. Hutcheson IR, Knowlden JM, Madden TA, et al. Estrogen receptor-mediated modulation of the EGFR/MAPK pathway in tamoxifen-resistant MCF-7 cells. Breast Cancer Res Treat 2003;81:81-93.

15. Jordan NJ, Gee JM, Barrow D, et al. Increased constitutive activity of $\mathrm{PKB} / \mathrm{Akt}$ in tamoxifen resistant breast cancer MCF7 cells. Breast Cancer Res Treat 2004;87:167-80.

16. Kahlert S, Nuedling S, van Eickels M, et al. Estrogen receptor alpha rapidly activates the IGF-1 receptor pathway. J Biol Chem 2000;275:18447-53.

17. Kato S, Endoh H, Masuhiro Y, et al. Activation of the estrogen receptor through phosphorylation by mitogen activated protein kinase. Science 1995;270:1491-4.

18. Knowlden JM, Hutcheson IR, Barrow D, et al. IGF-1R and EGFR crosstalk in tamoxifen resistant breast cancer cells. Breast Cancer Res Treat 2003;82:S171.

19. Kushner PJ, Agard DA, Greene GL, et al. Estrogen receptor pathways to AP-1. J Steroid Biochem Mol Biol 2000;74:311- 
20. Yang X, Ferguson AT, Nass SJ, et al. Transcriptional activation of estrogen receptor alpha in human breast cancer cells by histone deacetylase inhibition. Cancer Res 2000;60:6890-4.

21. Yang X, Phillips DL, Ferguson AT, et al. Synergistic activation of functional estrogen receptor (ER)-alpha by DNA methyltransferase and histone deacetylase inhibition in human ERalpha-negative breast cancer cells. Cancer Res 2001;61:70259.

22. Miller TW, Hennessy BT, Gonzalez-Angulo AM, et al. Hyperactivation of phosphatidylinositol-3 kinase promotes escape from hormone dependence in estrogen receptor-positive human breast cancer. J Clin Invest 2010;120:2406-13.

23. Noh W, Kim Y, Kim M, et al. Activation of the mTOR signaling pathway in breast cancer and its correlation with the clinicopathologic variables. Breast Cancer Res Treat 2008;110:477-83.

24. Oliveira M, Navarro, A, De Mattos-Arruda L, et al. PI3K pathway (PI3KP) dysregulation and response to PAN$\mathrm{PI} 3 \mathrm{~K} / \mathrm{AKT} / \mathrm{mTOR} /$ dual PI3K-mTOR inhibitors (PI3KPI) in metastatic breast cancer (MBC) patients (Pts). J Clin Oncol 2002;30:abstract 509 .

25. Patel BS, Co WS, Donat C, et al. Repression of breast cancer cell growth by proteasome inhibitors in vitro: impact of mitogen-activated protein kinase phosphatase . Cancer Biol Ther 2015;16:780-9.

26. Mayer I. Role of mTOR inhibition in preventing resistance and restoring sensitivity to hormone-targeted and HER2-targeted therapies in breast cancer. Clin Adv Hematol Oncol 2013;v11:217-24.

27. Baselga J, Campone M, Piccart M, et al. Everolimus in postmenopausal hormone-receptor-positive advanced breast cancer. N Engl J Med 2012;366:520-9.

28. Baselga J, Semiglazov V, van Dam P, et al. Phase II randomized study of neoadjuvant everolimus plus letrozole compared with placebo plus letrozole in patients with estrogen receptorpositive breast cancer. J Clin Oncol 2009;27:2630-7.

29. Wolff AC, Lazar AA, Bondarenko I, et al. Randomized phase III placebo-controlled trial of letrozole plus oral temsirolimus as first-line endocrine therapy in postmenopausal women with locally advanced or metastatic breast cancer. J Clin Oncol 2013;31:195-202.

30. Bachelot T, Bourgier C, Cropet C, et al. Randomized phase II trial of everolimus in combination with tamoxifen in patients with hormone receptor-positive, human epidermal growth factor receptor 2-negative metastatic breast cancer with prior exposure to aromatase inhibitors: a GINECO study. J Clin Oncol 2012;30:2718-24.

31. Beck JT, Hortobagyi GN, Campone M, et al. Everolimus plus exemestane as first-line therapy in HR, HER2 advanced breast cancer in BOLERO-2. Breast Cancer Res Treat 2014;143:45967.

32. Royce M, Villanueva C, Ozguroglu M, et al. BOLERO-4: Phase 2 trial of first-line everolimus (EVE) plus letrozole (LET) in estrogen receptor-positive $(\mathrm{ER}+)$, human epidermal growth factor receptor 2-negative (HER2-) advanced breast cancer (BC). ESMO 2016 [Epub ahead of print].

33. Kornblum NS, Manola J, Klein P, et al: PrECOG 0102: A randomized, double-blind, phase II trial of fulvestrant plus everolimus or placebo in post-menopausal women with hormone receptor-positive, HER2-negative metastatic breast cancer resistant to aromatase inhibitor therapy. 2016 SABCS. Abstract S1-02.

34. Swain SM, Baselga J, Kim SB, et al. Pertuzumab, trastuzumab, and docetaxel in HER2-positive metastatic breast cancer. $\mathrm{N}$ Engl J Med 2015;372:724-34.

35. Kaufman B, Mackey JR, Clemens MR, et al. Trastuzumab plus anastrozole versus anastrozole alone for the treatment of postmenopausal women with human epidermal growth factor receptor 2-positive, hormone receptor-positive metastatic breast cancer: results from the randomized phase III TAnDEM study. J Clin Oncol 2009;27:5529-37.

36. Johnston S, Pippen J, Pivot X, et al. Lapatinib combined with letrozole versus letrozole and placebo as first-line therapy for postmenopausal hormone receptor-positive metastatic breast cancer. J Clin Oncol 2012;27:5538-46.

37. National Comprehensive Cancer Network Inc. NCCN clinical practice guidelines in oncology: breast cancer, version 2. Fort Washington: NCCN; 2015.

38. Dalton WS. The proteasome. Semin Oncol 2004;31:3-9.

39. Ludwig H, Khayat D, Giaccone G, et al. Proteasome inhibition and its clinical prospects in the treatment of hematologic and solid malignancies. Cancer 2005;104:1794-807.

40. Rajkumar SV, Richardson PG, Hideshima T, et al. Proteasome inhibition as a novel therapeutic target in human cancer. J Clin Oncol 2005;23:630-9.

41. Haagenson KK, Wu GS. The role of MAP kinases and MAP kinase phosphatase-1 in resistance to breast cancer treatment. Cancer Metastasis Rev 2010;29:143-9.

42. Orlowski RZ, Dees EC. The role of the ubiquitination-proteasome pathway in breast cancer: applying drugs that affect the ubiquitin-proteasome pathway to the therapy of breast cancer. Breast Cancer Res 2003;5:1-7.

43. Cardoso F, Ross JS, Picart MJ, et al. Targeting the ubiquitinproteasome pathway in breast cancer. Clin Breast Cancer 2004;5:148-57.

44. Thaler S, Thiede G, Hengstler JG, et al. The proteasome inhibitor Bortezomib (Velcade) as potential inhibitor of estrogen receptor-positive breast cancer. Int $\mathrm{J}$ Cancer 2015;137:686-97.

45. Adelson KB, Ramaswamy B, Sparano JA, et al. Randomized phase II trial of fulvestrant alone or in combination with bortezomib in hormone receptor-positive metastatic breast cancer resistant to aromatase inhibitors: a New York cancer consortium trial. Abstract S6-03. SABCS 2014

46. Spange S, Wagner T, Heinzel T, Krämer OH. Acetylation of non-histone proteins modulates cellular signalling at multiple levels. Int J Biochem Cell Biol 2009;41:185-98.

47. Chang HG, Kim SJ, Chung KW, et al. Tamoxifen-resistant breast cancers show less frequent methylation of the estrogen receptor beta but not the estrogen receptor alpha gene. J Mol Med 2005;83:132-9.

48. Raha P, Thomas S, Thurn KT, et al. Combined histone deacetylase inhibition and tamoxifen induces apoptosis in tamoxifenresistant breast cancer models, by reversing Bcl-2 overexpression. Breast Cancer Res 2015;17:26.

49. Sabnis GJ, Goloubeva OG, Kazi AA, et al. HDAC inhibitor entinostat restores responsiveness of letrozole resistant MCF7Ca xenografts to AIs through modulation of Her-2. Mol Cancer Ther 2013;12:10.

50. Yardley DA, Ismail-Khan R, Klein P, et al. Results of ENCORE 301, a randomized, phase II, double-blind, placebocontrolled study of exemestane with or without entinostat in postmenopausal women with locally recurrent or metastatic estrogen receptor-positive (ER+) breast cancer progressing on a nonsteroidal aromatase inhibitor (AI). J Clin Oncol 2011; suppl 27:abstr 268.

51. Müller BM, Jana L, Kasajima A, et al. Differential expression 
of histone deacetylases HDAC1, 2 and 3 in human breast cancer--overexpression of HDAC2 and HDAC3 is associated with clinicopathological indicators of disease progression. BMC Cancer 2013;13:215.

52. Heer K, Kumar H, Read JR, et al. Serum vascular endothelial growth factor in breast cancer: its relation with cancer type and estrogen receptor status. Clin Cancer Res 2001;7:3491-4.

53. Gasparini G. Prognostic value of vascular endothelial growth factor in breast cancer. Oncologist 2000;5:37-44.

54. Yardley DA, Burris HA 3rd, Clark BL, et al. Hormonal therapy plus bevacizumab in postmenopausal patients who have hormone receptor-positive metastatic breast cancer: a phase II Trial of the Sarah Cannon Oncology Research Consortium. Clin Breast Cancer 2011;11:146-52.

55. Martín M, Loibl S, von Minckwitz G. Phase III trial evaluating the addition of bevacizumab to endocrine therapy as first-line treatment for advanced breast cancer: the letrozole/fulvestrant and avastin (LEA) study. J Clin Oncol 2015;33:1045-52.

56. Jacquemier J, Adelaide J, Parc P, et al. Expression of the FGFR1 gene in human breast-carcinoma cells. Int $\mathrm{J}$ Cancer 1994;59:373-8.

57. Turner N, Pearson A, Sharpe R, et al. FGFR1 amplification drives endocrine therapy resistance and is a therapeutic target in breast cancer. Cancer Res 2010;70:2085-94.

58. Johnson DE, Williams LT. Structural and functional diversity in the FGF receptor multigene family. Adv Cancer Res 1993;60:1-41.

59. Haugsten EM, Wiedlocha A, Olsnes S, et al. Roles of fibroblast growth factor receptors in carcinogenesis. Mol Cancer Res 2010;8:1439-52.

60. Penault-Llorca F, Bertucci F, Adelaide J et al. Expression of FGF and FGF receptor genes in human breast cancer. Int J Cancer 1995;61:170-6.

61. André F, Bachelot T, Campone M, et al. Targeting FGFR with dovitinib (TKI258): preclinical and clinical data in breast cancer. Clin Cancer Res 2013;19:3693-702.

62. Gozgit JM, Wong MJ, Moran L, et al. Ponatinib (AP24534), a multitargeted pan-FGFR inhibitor with activity in multiple FGFR-amplified or mutated cancer models. Mol Cancer Ther 2012;11:690-9.

63. Andre F, Neven P, Musolino A, et al. Dovitinib plus fulvestrant in postmenopausal endocrine resistant HER2-/HR + breast cancer: a phase II, randomized, placebo-controlled study. ASCO Meeting Abstracts 2013;31:TPS651.

64. Miller TW, Balko JM, Fox EM, et al. ER $\alpha$-dependent E2F transcription can mediate resistance to estrogen deprivation in human breast cancer. Cancer Discov 2011;1:338-51.

65. Dean JL, Thangavel C, McClendon AK, et al. Therapeutic CDK4/6 inhibition in breast cancer: key mechanisms of response and failure. Oncogene 2010;29:4018-32.

66. Shapiro GI. Cyclin-dependent kinase pathways as targets for cancer treatment. J Clin Oncol 2006;24:1770-83.

67. Santo L, Siu KT, Raje N. Targeting cyclin-dependent kinases and cell cycle progression in human cancers. Semin Oncol 2015;42:788-800.

68. Turner NC, Ro J, André F, et al. Palbociclib in hormone-receptor-positive advanced breast cancer. $N$ Engl J Med 2015;373:209-19.
69. Finn RS, Crown JP, Lang I, et al. The cyclin-dependent kinase $4 / 6$ inhibitor palbociclib in combination with letrozole versus letrozole alone as first-line treatment of oestrogen receptorpositive, HER2-negative, advanced breast cancer (PALOMA1/TRIO-18). A randomised phase 2 study. Lancet Oncol 2015;16:25-35.

70. Finn RS, Martin M, Rugo HS, et al. PALOMA-2: Primary results from a phase III trial of palbociclib $(\mathrm{P})$ with letrozole (L) compared with letrozole alone in postmenopausal women with ER+/HER2- advanced breast cancer. J Clin Oncol 2016;suppl 34:abstr 507.

71. Turner NC, Ro J, Andre F, et al. PALOMA3: A double-blind, phase III trial of fulvestrant with or without palbociclib in preand post-menopausal women with hormone receptor-positive, HER2-negative metastatic breast cancer that progressed on prior endocrine therapy. Abstract J Clin Oncol 2015;33(15 suppl).

72. Montagna E, Bagnardi V, Viale G, et al. Changes in PgR and $\mathrm{Ki}-67$ in residual tumour and outcome of breast cancer patients treated with neoadjuvant chemotherapy. Ann Oncol 2015;26:307-13.

73. Ma CX, Gao F, Northfelt D, et al. A phase II trial of neoadjuvant palbociclib, a cyclin-dependent kinase 4/6 inhibitor, in combination with anastrozole for clinical stage 2 or 3 estrogen receptor positive HER2 negative breast cancer. 2015 SABCS. Abstract S6-05.

74. Hurvitz S, Abad MF, Rostorfer R, et al. Interim results from neoMONARCH: A neoadjuvant phase II study of abemaciclib in postmenopausal women with HR + /HER2- breast cancer (BC). Ann Oncol 2016;27(suppl 6).

75. Hortobagyi GN, Stemmer SM, Burris HA, et al. First-line ribociclib + letrozole for postmenopausal women with hormone receptor-positive (HR+), HER2-negative (HER2-), advanced breast cancer (ABC). 2016 ESMO. Abstract LBA1_PR.

76. ClinicalTrials.gov. Study of Efficacy and Safety of LEE011 in Men and Postmenopausal Women With Advanced Breast Cancer. (MONALEESA-3) NCT02422615. Available from: https://clinicaltrials.gov/ct2/show/NCT02422615

77. Tolaney SM, Rosen LS, Beeram M, et al. Clinical activity of abemaciclib, an oral cell cycle inhibitor, in metastatic breast cancer. Cancer Res 2015;75:P5-19-13.

78. Robertson JF, Lindemann JP, Llombart-Cussac A, et al. Fulvestrant $500 \mathrm{mg}$ versus anastrozole $1 \mathrm{mg}$ for the first-line treatment of advanced breast cancer: follow-up analysis from the randomized 'FIRST' study. Breast Cancer Res Treat 2012;136:503-11.

79. Robertson JFR, Llombart-Cussac A, Feltl D, et al. Fulvestrant $500 \mathrm{mg}$ versus anastrozole as first-line treatment for advanced breast cancer: Overall survival from the phase II "first" study. Presented at 2014 San Antonio Breast Cancer Symposium; December 9-13, 2014; San Antonio, Texas. Abstract S6-04.

80. Ellis MJ, Bondarenko I, Trishkina E, et al. FALCON: A phase III randomised trial of fulvestrant $500 \mathrm{mg}$ vs anastrozole for hormone receptor-positive advanced breast cancer. Abstract LBA14_PR. ESMO 2016. 